\title{
Microscopy on Drugs: Characterization and Quantification of Pt-based Pharmaceuticals using the STEM.
}

Alexandra A. Sheader ${ }^{1 *}$, Gema Vizcay-Barrena ${ }^{2}$, Roland A. Fleck ${ }^{2}$, Sarah J. L. Flatters ${ }^{3}$, Peter D. Nellist ${ }^{1}$

1. Department of Materials, University of Oxford, UK

2. Centre For Ultrastructural Imaging, King's College London, UK

3. Wolfson Centre for Age-Related Diseases, Institute of Psychiatry, Psychology and Neuroscience, King's College London, UK

${ }^{*}$ Corresponding author: alex.sheader@materials.ox.ac.uk

High-angle annular dark field (HAADF) scanning transmission electron microscopy (STEM) is a powerful method for imaging heavy elements. In modern aberration-corrected instruments, very high spatial resolution can, with careful consideration given to the experimental setup, be combined with fully quantitative imaging where image contrast depends only on atomic number and thickness. Advances in HAADF imaging and simulation mean it is now routinely possible to characterize samples with atomcounting errors as small as \pm 1 atom [1].

While such approaches have most commonly been applied to inorganic specimens such as catalysts [2], quantitative HAADF imaging techniques have recently been used to examine the nanoscale structure of the platinum-based chemotherapy drugs cisplatin and oxaliplatin [3]. Such medicines frequently play key roles in the first line treatment of cancer. These platinum-containing chemotherapeutics are used in the treatment of several different cancers, including testicular, ovarian, and head and neck cancer.

However, both drugs are notorious for inducing particularly unpleasant side-effects in patients, not least of which is chronic and often irreversible nerve dysfunction which cannot be alleviated with analgesics [4]. Chemotherapy-induced peripheral neuropathy (CIPN) therefore presents a serious clinical challenge, and affects quality of life and can impact on patient survival. There is therefore a pressing need to understand how these drugs behave inside cells, particularly in the sensory nerves which are known sites of platinum accumulation and mitochondrial dysfunction following treatment [5].

Following rapid dissection of the dorsal root ganglia of rats treated with oxaliplatin, sections were prepared for STEM investigation using cryoultramicrotomy. We use a continuous-acquisition approach while collecting energy-dispersive $\mathrm{x}$-ray spectroscopy data as a strategy to minimize electron beam-induced damage, which allows rapid identification of promising Pt-based candidates within a tissue section.

Quantitative HAADF imaging in the STEM is then used to perform highly localized and sensitive 'weighing' of platinum, and clusters and individual atoms of platinum are visualized and characterized within these cells. Careful comparison with simulation confirms the platinum nature of the atoms imaged, and allows for quantification of oxaliplatin content from cell to cell [6].

References:

[1] K H W van den Bos et al., Advances in Physics: X 3 (2018), p. 1480420. https://doi.org/10.1080/23746149.2018.1480420 (accessed Feb 5th, 2019) 
[2] AM Varambhia et al., Particle \& Particle Systems Characterization 33 (2016), p. 438.

[3] AA Sheader et al., Journal of Microscopy 270 (2018), p. 92.

[4] MSeretny et al., PAIN 155 (2014), p. 2461.

[5] SJL Flatters, PM Dougherty, LA Colvin, British Journal of Anaesthesia 119 (2017), p. 737.

[6] The authors acknowledge use of the South of England Analytical Electron Microscope (EP/K040375/1), within the David Cockayne Centre for Electron Microscopy, Department of Materials, University of Oxford, alongside financial support provided by the Henry Royce Institute (Grant ref EP/R010145/1). This research was supported by DTP studentship awarded by the University of Oxford Department of Materials (AAS), and a Physiological Society Research Grant 2015 awarded to SJLF.

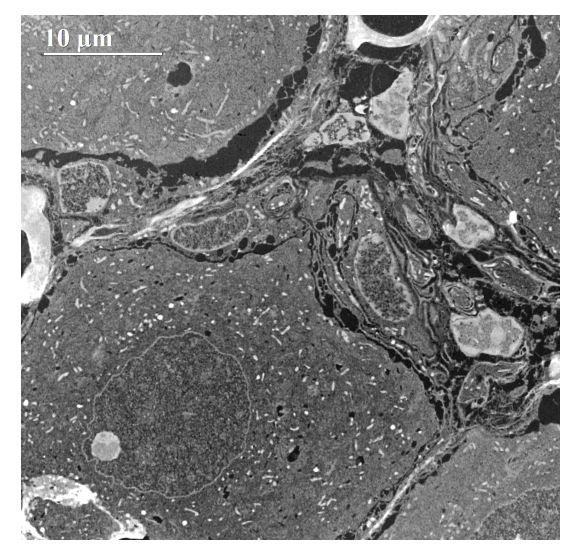

Figure 1. Example of low-magnification HAADF image obtained on the JEOL ARM 200F instrument of a cryosectioned rat Dorsal Root Ganglion.

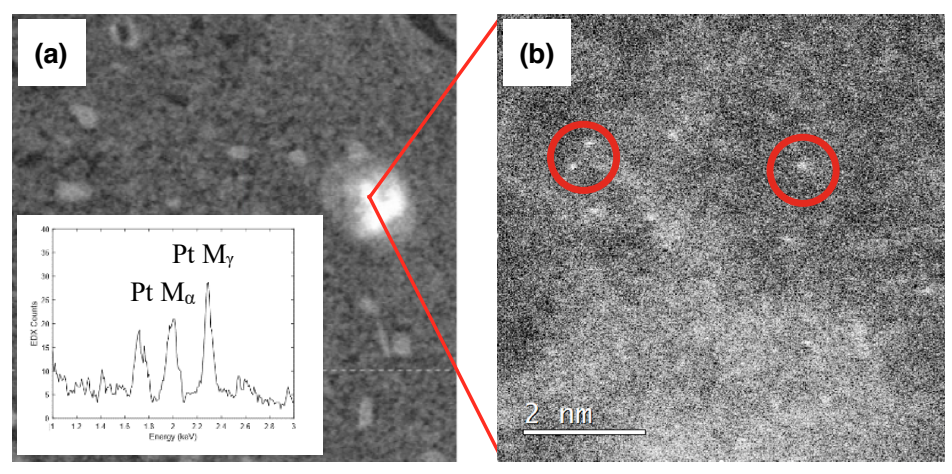

Figure 2. (a) Feature within a cell is first identified using continuously-acquired EDX (inset) before (b) increased magnification shows individual atoms suitable for quantification. 